Revue internationale P.M.E.

Économie et gestion de la petite et moyenne entreprise

\title{
Performance industrielle et contribution des sous-traitants nationaux : analyse du secteur aérospatial canadien dans le contexte nord-américain
}

\section{Mario Bourgault}

Volume 11, numéro 1, 1998

URI : https://id.erudit.org/iderudit/1009035ar

DOI : https://doi.org/10.7202/1009035ar

Aller au sommaire du numéro

Éditeur(s)

Presses de l’Université du Québec

ISSN

0776-5436 (imprimé)

1918-9699 (numérique)

Découvrir la revue

Citer cet article

Bourgault, M. (1998). Performance industrielle et contribution des sous-traitants nationaux : analyse du secteur aérospatial canadien dans le contexte nord-américain. Revue internationale P.M.E., 11(1), 41-63. https://doi.org/10.7202/1009035ar
Résumé de l'article

L'industrie aérospatiale présente des défis importants pour les PME impliquées à titre de sous-traitants. La prédominance des facteurs technologiques et de la concurrence internationale impose le développement d'une gamme élargie de compétences. Cet article présente les résultats d'une recherche empirique visant à identifier ces compétences. Les données sont tirées d'une enquête réalisée auprès de 273 petites et moyennes entreprises localisées sur le territoire nord-américain (Canada et États-Unis) et impliquées dans ce secteur. Les résultats montrent des écarts importants entre les firmes des deux pays, en particulier chez les firmes de taille moyenne. Les PME canadiennes s'écartent peu du profil de sous-traitant traditionnel alors que les firmes américaines affichent un comportement nettement plus proactif, autant sur le plan organisationnel que technologique. Étant donné le succès récent de plusieurs donneurs d'ordres canadiens sur les marchés étrangers, les résultats de cette étude soulèvent de nombreuses interrogations quant à la capacité des firmes sous-traitantes à contribuer à ce succès. 


\title{
Performance industrielle et contribution des sous-traitants nationaux: analyse du secteur aérospatial canadien dans le contexte nord-américain
}

\author{
Mario BOURGAULT \\ École polytechnique de Montréal
}

\author{
MOTS CLÉS
}

\section{Sous-traitance - Industrie aérospatiale \\ Petite et moyenne entreprise (PME) - Compétences \\ Performance - Grappe industrielle}

\begin{abstract}
RÉSUMÉ
L'industrie aérospatiale présente des défis importants pour les PME impliquées à titre de sous-traitants. La prédominance des facteurs technologiques et de la concurrence internationale impose le développement d'une gamme élargie de compétences. Cet article présente les résultats d'une recherche empirique visant à identifier ces compétences. Les données sont tirées d'une enquête réalisée auprès de 273 petites et moyennes entreprises localisées sur le territoire nord-américain (Canada et États-Unis) et impliquées dans ce secteur. Les résultats montrent des
\end{abstract}

\section{L'AUTEUR}

Mario Bourgault détient un doctorat (Ph.D.) en génie industriel (orientation gestion de la technologie). II est actuellement chercheur au Département de mathématiques et de génie industriel de l'École polytechnique de Montréal. II est également associé au CIRANO et à la Chaire Jarislowsky. Ses domaines d'enseignement et de recherche touchent l'analyse des rapports interfirmes, la gestion de projets, de même que la gestion de la technologie et de l'innovation. Adresse de correspondance: École polytechnique, Département de mathématiques et de génie industriel, C.P. 6079, succ. Centre-ville, Montréal, Québec, Canada, H3C 3A7. Courriel: bourgaum@cirano, umontreal.ca. 
écarts importants entre les firmes des deux pays, en particulier chez les firmes de taille moyenne. Les PME canadiennes s'écartent peu du profil de sous-traitant traditionnel alors que les firmes américaines affichent un comportement nettement plus proactif, autant sur le plan organisationnel que technologique. Étant donné le succès récent de plusieurs donneurs d'ordres canadiens sur les marchés étrangers, les résultats de cette étude soulèvent de nombreuses interrogations quant à la capacité des firmes sous-traitantes à contribuer à ce succès.

\begin{abstract}
The aerospace industry is most challenging for small and medium enterprises (SMEs) which are involved as subcontractors. New conditions such as globalization and technology-led competition bring about major changes in terms of which capabilities are required to sustain corporate growth. This paper offers an overview of a survey conducted in North America where 273 SMEs from Canada and the United States were evaluated and compared. Results indicate significant differences between those two countries and particularly for the medium-sized firms. Canadian subcontractors tend to maintain a traditional profile whereas their counterparts from United States are clearly more proactive both from a technological and organizational perspective. In view of Canadian major firms' success on international markets, these results raise questions about the capacity of the Canadian supplier base to participate in building and reinforcing this success.
\end{abstract}

\title{
RESUMEN
}

El sector aerospacial presenta desafíos importantes para las pequeñas y medianas empresas (PyMEs) implicadas como subcontratistas. La globalización y la predominancia de factores tecnológicos generan cambios mayores en el ambiente competitivo de las empresas, forzándolas a desarrollar una gama cada día más amplia de competencias para poder sostener el crecimiento y la rentabilidad. Este artículo presenta los resultados de una investigación empírica en la cual 273 PME canadienses y estadounidenses fueron comparadas y evaluadas por medio de una encuesta. Los resultados muestran diferencias significativas entre las empresas de los dos países, en particular en las firmas de talla mediana. Los subcontratistas canadienses muestran un comportamiento más tradicional mientras que los estadounidenses son claramente más proactivas, tanto desde el punto de vista tecnológico como desde el punto de vista organizacional. Estos resultados ponen de relieve el éxito reciente de varias grandes empresas canadienses y cuestionan la capacidad de la base de proveedores para construir y compartir este éxito.

\section{Introduction}

Comme la concurrence mondiale est de plus en plus fondée sur la prédominance des connaissances et de l'innovation, les secteurs industriels à forte intensité technologique font l'objet d'une attention particulière de la part des responsables de politiques industrielles. Du point de vue gouvernemental, on souhaite voir émerger des industries à haute valeur ajoutée et favoriser la croissance de celles qui sont 
déjà concurrentielles sur le plan international. En raison toutefois de l'incertitude croissante liée à l'évolution des systèmes économiques et technologiques, la mise en place de ces politiques demeure complexe et leur nature même est en constante évolution (Saviotti, 1995). Dans la mesure de leurs moyens, certains gouvernements tentent de favoriser la concertation par l'introduction de diverses mesures, comme ce fut le cas récemment au Québec avec la politique des grappes industrielles, laquelle s'est inspirée des filières françaises. Cette approche a mené à la création de nouveaux regroupements d'acteurs industriels, notamment dans les secteurs de l'aérospatiale, de l'énergie et de la pharmaceutique.

Une des motivations sous-jacentes à ce type d'interventions est liée à la création de liens entre les différentes firmes formant le tissu industriel. Tout en reconnaissant le rôle central joué par les grandes firmes, les gouvernements cherchent à maximiser les retombées technologiques et économiques sur les nombreuses PME agissant comme sous-traitants. Cette position est largement justifiée lorsque l'on considère le rôle prépondérant que jouent ces dernières dans la plupart des économies (Julien et al., 1997).

La qualité de la base de sous-traitance nationale, thème central de la présente recherche, doit par ailleurs jouer un rôle dans la façon et dans l'efficacité avec lesquelles les rapports entre PME et grandes firmes se concrétisent. Dans son modèle des déterminants de l'avantage concurrentiel, Porter (1990) soutient que le développement d'industries fortes repose entre autres sur la présence d'industries de soutien (related and supporting industries). L'existence d'une base de soustraitance forte conférerait un avantage aux grandes entreprises, tant au regard de la coordination des opérations qu'à celui de l'exécution des activités de développement. La réalisation de projets avec des entreprises nationales dominantes serait de nature à stimuler la croissance des firmes sous-traitantes qui pourraient, à leur tour, devenir des firmes de classe mondiale. Une interaction continue de part et d'autre faciliterait donc la croissance de l'industrie dans son ensemble.

Le modèle de Porter a suscité de nombreuses critiques d'auteurs issus d'économies de moindre envergure que celle des États-Unis (Jacobs et De Jong, 1992). La pertinence du modèle appliqué au Canada et au Québec a également été mise en doute (Demers et Hafsi, 1993) ; pour ces auteurs, les grandes entreprises seraient plus enclines à «créer leur propre réseau international qu'à profiter de leur base nationale». Rugman et O'Cruz (1993) ont, pour leur part, mis en doute l'applicabilité de ce modèle à la situation canadienne, étant donné les nombreux échanges existant entre le Canada et les États-Unis. Cette position se vérifie dans des secteurs manufacturiers traditionnels comme celui de l'automobile où les échanges entre filiales situées de chaque côté de la frontière constituent une bonne partie des exportations canadiennes. Elle soulève, par ailleurs, la même question pour des industries dont l'essor est plus récent comme c'est le cas de l'aérospatiale. Ce secteur affiche actuellement une grande vitalité grâce, entre autres, aux succès 
répétés de grandes entreprises nationales comme Bombardier, CAE et Spar Aérospatiale. Il s'avère pertinent d'évaluer si ce succès est aussi le reflet des firmes en périphérie des grands donneurs d'ordres, soit les sous-traitants.

L'objet de la présente recherche consiste à dresser le profil des PME soustraitantes de l'industrie aérospatiale canadienne en tenant compte des exigences mondiales actuelles. Les questions sous-jacentes à cette étude touchent la capacité des PME à soutenir les grandes entreprises à succès et à accroître, ce faisant, leur propre niveau de compétences. De façon plus précise, la recherche vise à répondre aux questions suivantes :

- Quel est le profil des PME sous-traitantes au regard des compétences technologiques et organisationnelles? Ces compétences sont-elles des facteurs explicatifs importants de la performance des firmes?

- Étant donné les liens étroits entre l'industrie canadienne et l'industrie américaine, quelle comparaison peut-on faire entre les sous-traitants canadiens et les américains?

- La taille influe-t-elle sur la performance des PME sous-traitantes œuvrant dans cette industrie?

Ces questions présentent un intérêt vital compte tenu des changements qui s'opèrent depuis quelques années dans l'environnement des grandes firmes. Non seulement l'ouverture des marchés permet-elle de transiger plus facilement avec des firmes extérieures, mais en outre les nouveaux moyens de communication et de traitement de l'information fournissent aux grands donneurs d'ordres de plus en plus d'outils pour élargir leur base de sous-traitance à l'international (Bergeron et Raymond, 1996).

Le cas de l'industrie aérospatiale canadienne constitue un terrain propice pour évaluer la qualité des sous-traitants nationaux œuvrant en périphérie des grands constructeurs. Bien que modeste par rapport aux géants américains et européens, cette industrie a su se tailler une place enviable sur les marchés internationaux. En faisant abstraction de la récession des années 1991-1993, les statistiques indiquent une augmentation totale des ventes de l'ordre de $140 \%$ au cours de la période 1986-1996. Aussi, les données préliminaires portant sur l'année 1998 indiquent que l'industrie pourrait atteindre un niveau de ventes de 14,5 milliards dollars canadiens, passant ainsi du sixième au quatrième rang mondial, devant l'Allemagne et le Japon ${ }^{1}$. Les marchés étrangers occupent par ailleurs une place de plus en plus grande, représentant, au terme de la décennie 1986-1996, plus de $75 \%$ de l'ensemble des ventes.

1. Les premières places sont occupées par les États-Unis, la France et la Grande-Bretagne. Les ventes prévues de l'industrie aérospatiale américaine, en 1997, seraient de l'ordre de 130 milliards de dollars américains (AIAA, 1997). 
L'industrie canadienne comporte d'autres particularités intéressantes, notamment le fait que bon nombre de grandes entreprises sont des filiales de compagnies étrangères. Dans la liste des 20 plus importantes firmes canadiennes de l'aérospatiale dressée par Industrie Canada (1996a), au moins 15 demeurent sous contrôle étranger ${ }^{2}$. Aussi, la part réelle des retombées relativement aux contrats octroyés à des sous-traitants nationaux demeure difficile à évaluer ${ }^{3}$. Même si de sérieux efforts sont faits chez certains constructeurs pour «acheter canadien », il est évident que la forte proportion de firmes étrangères installées au Canada joue un rôle dans la répartition des contrats de sous-traitance, car les politiques d'achat sont souvent établies par les maisons mères. Les statistiques montrent qu'au total, à tout le moins dans le secteur de la construction d'avions, le Canada est déficitaire et qu'une part importante des biens et des services intermédiaires sont importés, notamment par des transactions intrafirmes (Industrie Canada, 1995).

\section{Cadre d'analyse: Les compétences des sous-traitants}

La présente recherche propose une évaluation des sous-traitants sous l'angle des compétences. Il s'agit en fait d'identifier et de mesurer un certain nombre de caractéristiques organisationnelles et d'établir des comparaisons entre les firmes concernées selon leur situation géographique et leur taille. La recherche vise aussi à mesurer l'importance réelle des compétences technologiques et organisationnelles dans la performance des sous-traitants.

L'analyse des compétences et des ressources de la firme fait aujourd'hui l'objet de nombreuses recherches. Dans le domaine de la stratégie, par exemple, on y voit un substitut intéressant à l'analyse dominante des dernières années qui a surtout été orientée vers l'environnement de la firme (Doz, 1994). La diffusion rapide du thème des «core competencies » dans le domaine de la gestion constitue également une illustration frappante de cet intérêt. La présente recherche se situe donc dans le courant actuel qui préconise le recours à plusieurs dimensions internes pour mieux refléter la complexité de la firme.

2. Dans le secteur de la construction d'avions, les données de 1992 établissent à $48 \%$ la proportion d'actifs contrôlés par les étrangers, ce qui équivaut à $55,4 \%$ de la valeur produite (Industrie Canada, 1995).

3. Ces données sont gểnéralement confidentielles et donc difficiles à obtenir. Dans leur étude de Pratt \& Whitney Canada, DeBresson et al. (1991) ont estimé le contenu étranger des produits de cette firme entre $15 \%$ et $50 \%$, selon la source consultée. Dans le secteur de la construction d'avions, Industrie Canada (1995) estime que $40 \%$ de l'ensemble des biens et services achetés par les fabricants de l'industrie le sont à l'étranger. Aucune distinction n'est toutefois faite quant à la nationalité des entreprises concernées. 
Les divers travaux consacrés aux relations entre sous-traitants et donneurs d'ordres ont, par ailleurs, montré qu'il existe plusieurs facteurs pouvant influencer le succès de ces rapports interfirmes. Plusieurs études ont, par exemple, mis en évidence la différence existant entre les méthodes nord-américaines et les méthodes japonaises (Helper et Sako, 1995 ; Kamath et Liker, 1994). De façon plus précise, Dyer et Ouchi (1993) ont identifié certains aspects des relations caractérisant le partenariat type entre les donneurs d'ordres japonais et leurs fournisseurs : mise en commun des efforts pour définir les besoins et résoudre les problèmes ; communication fréquente et planifiée; partage d'information constant ; investissement adapté aux besoins des clients ; flexibilité dans les ententes contractuelles pour refléter les fluctuations économiques. L'implication des donneurs d'ordres peut donc jouer un rôle sur la performance des sous-traitants.

L'approche théorique de cette recherche repose donc sur deux propositions :

Proposition 1 : Dans une industrie de haute technologie telle que l'aérospatiale, les PME sous-traitantes doivent acquérir une gamme élargie de compétences (technologiques et organisationnelles) dont l'impact présumé sur leur performance est positif.

La prédominance du facteur technologique dans l'industrie aérospatiale force les PME à acquérir ou à développer une large gamme de compétences. Sur le plan technologique, ce processus s'opère d'abord par l'entremise d'activités de recherche et développement (R-D) qui permettent d'améliorer les connaissances et les savoirfaire et, de façon plus particulière, les produits et les procédés (Sen et Rubenstein, 1990; Morbey, 1988). Par de tels investissements, la firme accroît à la fois son potentiel d'apprentissage à l'interne et sa capacité d'absorber les connaissances à l'externe. Les activités de recherche et développement doivent, par ailleurs, être soutenues par une veille technologique constante de manière à pouvoir identifier les occasions d'affaires, à comprendre et à prévoir les actions des compétiteurs, et, surtout, à pouvoir évaluer les technologies émergentes potentiellement intéressantes (Jacob, Julien et Raymond, 1997; Van Wyk, 1997). Au-delà des compétences de création et de reconnaissance des occasions d'affaires, la PME sous-traitante doit être en mesure de satisfaire aux normes extrêmement sévères de fabrication dictées par l'industrie. Elle y parviendra en adoptant une série de technologies (hard et soft) couvrant un spectre relativement large d'applications, que ce soit au regard de la conception et de l'ingénierie, de la fabrication et de l'assemblage, du contrôle de qualité, de la manutention des stocks ou à celui de la planification des ressources. De nombreux chercheurs ont étudié l'impact de ces technologies et plusieurs ont établi un lien étroit entre leur adoption et la capacité des firmes à accroître leur efficience en ce qui concerne le coût, la qualité, la flexibilité et les délais de livraison (Bessant, 1993; Meredith, 1988). 
Dans un contexte de spécialisation croissante, les sous-traitants qui veulent faire affaire avec les grands donneurs d'ordres doivent globalement compter sur des compétences techniques et des savoir-faire uniques. De façon générale, il est reconnu que les compétences techniques des employés sont devenues un préalable à l'introduction d'innovations au sein des firmes, et tout spécialement les technologies de production (Lefebvre, Harvey et Lefebvre, 1991). Il en va de même pour le processus de développement de nouveaux produits : là encore, le succès est souvent relié à la qualité des équipes techniques de conception et de production (Schewe, 1994 ; Zirger et Maidique, 1990). L'avantage tiré d'un savoir-faire lié à certains produits constitue également un actif important pour les firmes, surtout pour les plus petites qui évoluent au sein d'industries hautement concurrentielles.

Sur le plan organisationnel, les PME sous-traitantes doivent aussi démontrer une large gamme de compétences de façon à résister aux nombreuses pressions exercées sur elles (Presutti, 1991). La qualité de la gestion apparaît donc essentielle, tout comme les efforts en matière commerciale. À cet égard, les PME doivent de plus en plus orienter leur développement vers l'exploitation des marchés internationaux, ce qui apparaît encore difficile à réaliser dans de nombreux cas (Bonaccorsi, 1992). Tout en cherchant à développer de nouveaux marchés, les sous-traitants doivent également soutenir leur clientèle en dépit des difficultés créées par une réduction généralisée de la base de sous-traitance des grands donneurs d'ordres. Compte tenu des nombreuses responsabilités que les donneurs d'ordres leur imposent, les sous-traitants sont sélectionnés avec une attention vigilante et leur « carnet de route» revêt une importance considérable. Du point de vue de l'entreprise sous-traitante, le maintien d'une image de marque constitue donc une arme puissante pour lutter contre la réduction généralisée des bases de sous-traitance que s'imposent la plupart des donneurs d'ordres. La réputation de la firme peut ainsi représenter un levier efficace dans la mesure où la firme est capable de perpétuer l'opinion favorable des marchés à son égard (Hall, 1993). Par ailleurs, une plus grande stabilité dans les relations commerciales exige des fournisseurs une performance soutenue sur le plan financier (Oakey, 1994 ; Kolay, 1992).

Proposition 2: La relation entre compétences et performance des firmes sous-traitantes dépend de certaines caractéristiques de la relation entre sous-traitants et donneurs d'ordres.

Les caractéristiques de la relation entre sous-traitants et donneurs d'ordres constituent un volet essentiel pour une meilleure compréhension de la relation entre la performance des sous-traitants et leur gamme de compétences. Les sous-traitants sont actuellement soumis à des pressions qui les obligent à plus d'ouverture envers leurs clients. Aussi, le climat de coopération qu'on tente de plus en plus d'instituer entre les firmes tend à complexifier un certain nombre de décisions dans la mesure où le sous-traitant se trouve davantage lié aux besoins de son client et, par conséquent, en subit l'influence dans les choix qu'il doit poser (Hahn, Watts et Kim, 
1990). Le besoin accru de coordination interfirmes pour certaines activités exige une volonté commune d'échanger de l'information, et parfois même du personnel. Helper (1991) a ainsi montré les bénéfices à tirer, pour les deux parties, d'une position ouverte de résolution des problèmes et des conflits. Or, plusieurs études révèlent que les relations sont toujours perçues comme inéquitables et ce, même si les donneurs d'ordres affirment faire preuve de bonne volonté et poser des gestes concrets pour gagner la confiance de leurs sous-traitants. Les exigences des clients peuvent donc avoir un effet sur la performance globale des sous-traitants, et notamment sur la pertinence ou la contribution de leurs compétences (Stuart et McCutcheon, 1995). Le nouveau type de relation interfirmes modifie aussi le degré d'indépendance auquel les sous-traitants ont été longtemps habitués. En échange de l'engagement à long terme de leurs clients, les sous-traitants doivent souvent accepter une certaine forme «d'ingérence » de ces derniers. Or, ce changement peut être vécu difficilement, étant donné la crainte que le pouvoir accru du client éveille chez les sous-traitants (Kamath et Liker, 1990).

\section{Méthodologie de recherche}

Cette étude repose sur une enquête réalisée auprès de dirigeants d'entreprises soustraitantes de l'industrie aérospatiale nord-américaine. L'objectif étant de recueillir le plus grand nombre de données possible, le questionnaire est apparu l'outil le plus efficace. Ce questionnaire, auto-administré, fut donc posté directement aux dirigeants d'entreprises situées aux États-Unis et au Canada. L'échantillon final servant de base à la présente recherche comprend 273 firmes de moins de 500 employés. Sur l'ensemble des firmes visées par l'enquête et correspondant à cette définition de PME, ce nombre correspond à un taux de réponse fort satisfaisant de $23 \%$, comparable à des enquêtes du même type dans le milieu industriel (Liker et al., 1996; Swamidass, 1994).

L'ensemble des variables de l'enquête et comprises dans la présente analyse ont été validées théoriquement comme indiqué au tableau 1. Les mesures qui leur sont associées sont, quant à elles, fournies en annexe.

L'analyse empirique proposée dans cet article présente d'abord des comparaisons intergroupes pour chacune des variables considérées et ce, en fonction de la problématique présentée à la section précédente. Elle met en évidence certaines différences entre les PME selon la perspective choisie, c'est-à-dire selon la situation géographique et la taille. L'objectif principal de la recherche demeure toutefois la vérification d'hypothèses quant à la relation pouvant exister entre, d'une part, les variables indépendantes sélectionnées mesurant les compétences et, d'autre part, la performance des sous-traitants (propositions 1 et 2). Pour y arriver, l'approche préconisée est l'utilisation d'une méthode d'analyse multivariée classique : la régression multiple. 


\section{TABLEAU 1}

\section{Variables utilisées pour décrire les compétences et les relations interfirmes}

\begin{tabular}{|c|c|}
\hline Compétences technologiques & Justification théorique \\
\hline investissement en R-D & Sen et Rubenstein, 1990 ; Morbey, 1988 \\
\hline veille technologique & Jacob et al. 1997 ; Van Wyk, 1997 \\
\hline adoption de technologies avancées & Bessant, 1993 ; Meredith, 1988 \\
\hline compétence technique des employés & $\begin{array}{l}\text { Lefebvre } \text { et al. } 1991 \text {; Schewe, } 1994 \text {; Zirger } \\
\quad \text { et Maidique, } 1990\end{array}$ \\
\hline savoir-faire unique lié aux produits & Österlund, 1991 \\
\hline Compétences organisationnelles & Justification théorique \\
\hline habiletés de gestion & Schewe, 1994; Presutti, 1991 ; Helper, 1991 \\
\hline efforts de marketing & Paliwoda et Bonaccorsi, 1993 ; Dunn et al., 1991 \\
\hline stabilité financière & Kolay, 1992; Hahn et al., 1990 \\
\hline degré d'internationalisation des ventes & Bonaccorsi, 1992 \\
\hline réputation & De Brentani, 1989; Hall, 1993 \\
\hline maintien de réseaux fournisseurs et clients & Funk, 1993 ; Blenkhorn et Noori, 1990 \\
\hline Caractéristiques de la relation & Justification théorique \\
\hline degré d'exigence du donneur d'ordres & Stuart et McCutcheon, 1995 ; Han et al., 1993 \\
\hline degré de dépendance du sous-traitant & Kamath et Liker, 1990 \\
\hline influence du donneur d'ordres & Hahn et al., 1990; Lefebvre et al., 1990 \\
\hline
\end{tabular}

L'évaluation de cette performance a été établie en fonction des critères fixés par les clients (les donneurs d'ordres) lors d'une préenquête. Ainsi, le degré de performance des sous-traitants est établi en fonction de leur capacité à satisfaire à des exigences élevées de qualité, de coûts, de flexibilité de production et de délais de livraison. Le score de performance, obtenu par la moyenne de ces quatre facteurs (voir annexe), constitue donc cette variable dépendante à expliquer.

\section{Analyse des résultats}

Une première analyse descriptive des résultats permet de relever des différences significatives entre le profil moyen des PME sous-traitantes canadiennes et celui de leurs homologues américaines.

Des écarts sont d'abord relevés dans les variables mesurant les compétences technologiques ; c'est le cas des investissements en R-D qui sont plus importants chez les firmes canadiennes que chez les firmes américaines. Ce résultat, surprenant à première vue, doit être interprété à la lumière du contexte particulier des deux pays en ce qui a trait aux politiques de financement des activités de R-D. Aux 
États-Unis, le financement public des activités de R-D liées à l'aérospatiale et à la défense est particulièrement élevé : il représente près de $70 \%$ des sommes investies (Industrie Canada, 1996b). Pour diverses raisons qui sont autant d'ordre économique que politique, les investissements publics canadiens en R-D sont beaucoup plus modestes (de l'ordre de 1 milliard de dollars), mais la part assumée par les entreprises est nettement plus élevée, soit entre $75 \%$ et $80 \% 4$. Les entreprises canadiennes investissent donc une plus grande part de leur volume d'affaires dans les activités de R-D : ce fait semble aussi confirmé chez les PME sous-traitantes. L'écart observé entre les deux pays s'explique peut-être aussi par les politiques particulièrement favorables du Canada à l'égard des crédits d'impôt à la R-D. Les firmes canadiennes se distinguent non seulement par le taux affiché en R-D, mais aussi par l'importance de leurs activités de veille technologique. Ces résultats concordent dans la mesure où il s'agit d'activités complémentaires : une connaissance aiguë des possibilités et des besoins technologiques du milieu devrait en effet se traduire par une intensification des activités de R-D à l'interne.

Les résultats du tableau 2 indiquent de plus que les firmes américaines se distinguent de façon significative par le taux d'adoption des technologies avancées, ce qui confirme les données de l'OCDE (1995) sur la lenteur de la conversion des entreprises canadiennes aux nouvelles technologies. Il est intéressant d'explorer plus à fond ces différences et d'identifier le taux d'adoption de chaque technologie considérée. La figure 1 présente ces technologies une à une selon les taux d'adoption de l'échantillon étudié. Ainsi, les firmes sous-traitantes américaines ressortent par leur utilisation intensive des technologies et des méthodes de gestion du flux des matériaux et de l'inventaire, telles que les systèmes MRP I-II, la technologie des codes zébrés, le JIT, la gestion automatisée de l'inventaire de même que la manutention automatisée. Les entreprises canadiennes accusent en revanche un retard important au regard du contrôle statistique de la qualité et des procédés (SQC/SPC).

La différence entre les deux pays semble toutefois s'estomper en ce qui regarde les technologies de fabrication et d'ingénierie. En effet, le test statistique ne permet pas d'établir de différence significative entre les degrés d'adoption des technologies de $\mathrm{CAO}$, de CAO/FAO ou de DNC. Ces résultats confirment des études

4. La taille du Canada explique évidemment une bonne partie de l'écart de financement (en valeur absolue) entre les deux pays, mais il faut rappeler que des choix politiques importants ont modifié l'orientation de l'industrie aérospatiale au cours des décennies. Après la Seconde Guerre mondiale, le Canada détenait une forte compétence en développement d'aéronefs civils et militaires. Le début des années 1960 marqua un tournant avec l'abandon du projet Avro Arrow. Au cours des années suivantes, l'industrie s'est résolument orientée vers les marchés civils et, ce faisant, a réduit les possibilités d'appui financier de la défense dont profite largement sa rivale américaine (Industrie Canada, 1995). 
similaires réalisées tant aux États-Unis qu'au Canada (Statistique Canada, 1995 ; Swamidass, 1994). Globalement, il semble que les firmes canadiennes impliquées dans l'aérospatiale disposent d'équipements comparables à ceux des firmes américaines pour entreprendre les tâches de conception et de fabrication ; elles seraient toutefois moins bien outillées pour assurer une gestion efficace de la qualité et des flux de matériaux, comme l'exigent de plus en plus les grandes entreprises. Au regard des autres variables mesurant les compétences technologiques, rappelons que les firmes américaines sont aussi en position de force quant à la détention d'un savoir-faire unique lié à des produits spécifiques (tableau 2), ce qui leur confère une plus grande autonomie sur les marchés.

TABLEAU 2

Profil moyen des PME sous-traitantes canadiennes et américaines

\begin{tabular}{|c|c|c|c|}
\hline & $\begin{array}{l}\text { Canada } \\
n_{1}=132\end{array}$ & $\begin{array}{c}\text { États-Unis } \\
\qquad n_{2}=141\end{array}$ & $\begin{array}{c}\text { Niveau de } \\
\text { signification } \\
\mathbf{p}^{1}\end{array}$ \\
\hline \multicolumn{4}{|l|}{ Compétences technologiques } \\
\hline investissement en R-D (\%) & 4,80 & 2,50 & $* * *$ \\
\hline veille technologique ${ }^{2}$ & 5,28 & 4,93 & $* *$ \\
\hline adoption de technologies avancées ${ }^{3}$ & 6,39 & 8,11 & $* *$ \\
\hline compétences techniques des employés ${ }^{2}$ & 5,34 & 5,16 & \\
\hline savoir-faire unique lié aux produits² & 4,90 & 5,30 & $*$ \\
\hline \multicolumn{4}{|l|}{ Compétences organisationnelles } \\
\hline habiletés de gestion ${ }^{2}$ & 4,86 & 5,19 & $* *$ \\
\hline efforts de marketing ${ }^{2}$ & 4,22 & 4,24 & \\
\hline stabilité financière ${ }^{2}$ & 4,89 & 4,86 & \\
\hline degré d'internationalisation des ventes (\%) & 38,64 & 10,56 & $* * * *$ \\
\hline réputation ${ }^{2}$ & 5,86 & 5,90 & \\
\hline stabilité des réseaux fournisseurs et clients ${ }^{2}$ & 5,08 & 4,89 & \\
\hline \multicolumn{4}{|l|}{ Caractéristiques de la relation } \\
\hline degré de dépendance (\%) & 25,0 & 37,6 & $* *$ \\
\hline degré d'exigence ${ }^{2}$ & 4,60 & 4,20 & $* * *$ \\
\hline degré d'influence ${ }^{2}$ & 3,00 & 2,93 & \\
\hline taille du sous-traitant (nombre d'employés) & 91 & 80 & \\
\hline
\end{tabular}

1. Niveau de signification établi par le test de Student : ${ }^{*} \mathrm{p}<0,10 ;{ }^{* *} \mathrm{p}<0,05 ;{ }^{* * *} \mathrm{p}<0,01 ; * * * * \mathrm{p}<0,001 ;$ test bilatéral.

2. Mesures perceptuelles établies à l'aide d'échelles Likert en sept points ; voir annexe.

3. Voir figure 1 pour la liste des technologies. 
FIGURE 1

Adoption de technologies avancées

$100 \% \quad 80 \% \quad 60 \% \quad 40 \% \quad 20 \%$

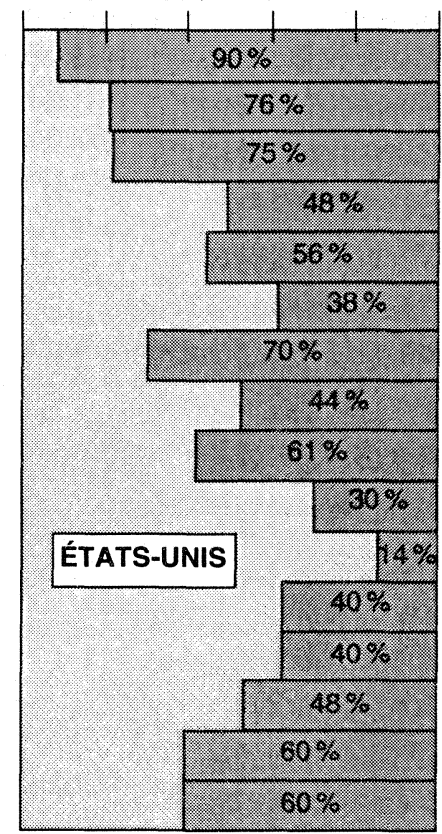

App. comptables

Prix de revient

Gest. inventaire

MRPI-II

Coût fab. Comm.

EDI

CAO

CAO/FAO intég.

CNC

DNC

Manut. Auto.

Codes barres

Contr. $Q$ info

JIT

SQC/SPC

Resp. des empl.
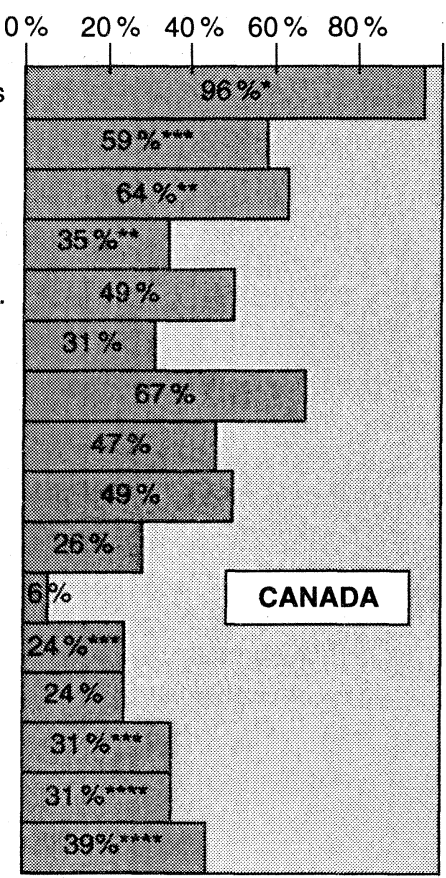

Proportion de firmes utilisant la technologie

Niveau de signification : ${ }^{*} \mathrm{p}<0,1 ;{ }^{* *} \mathrm{p}<0,05 ;{ }^{* * *} \mathrm{p}<0,01 ;{ }^{* * *} \mathrm{p}<0,001$

L'analyse des autres résultats du tableau 2 révèle deux différences marquées dans les compétences organisationnelles. Les firmes canadiennes démontrent des habiletés de gestion légèrement plus faibles que les firmes américaines, mais affichent un taux d'internationalisation des ventes beaucoup plus élevé $(38,64 \%$ contre $10,56 \%)$. Cet écart reflète bien la réalité rapportée par les statistiques officielles : en 1993, l'industrie canadienne exportait environ $70 \%$ de sa production contre $35 \%$ aux États-Unis. Un ratio moindre chez les sous-traitants était par ailleurs prévisible, vu que les exportations sont avant tout réalisées par les donneurs d'ordres.

\section{Effet de la taille des sous-traitants sur les compétences}

La firme sous-traitante est caractérisée non seulement par sa situation géographique, mais aussi par sa taille, dont nous avons cherché par la suite à mesurer les effets sur les variables utilisées. Cette seconde étape a été réalisée en constituant deux 
sous-groupes de taille différente pour chacun des deux pays ${ }^{5}$. Les sous-groupes ont été constitués à partir du calcul de la médiane sur la variable taille, pour l'ensemble de l'échantillon (voir tableau 3).

TABLEAU 3

Profil moyen des firmes sous-traitantes canadiennes et américaines, selon la taille

\begin{tabular}{|c|c|c|c|c|c|c|}
\hline & \multicolumn{3}{|c|}{ Firmes de petite taille $^{1}$} & \multicolumn{3}{|c|}{ Firmes de taille moyenne $^{1}$} \\
\hline & $\begin{array}{c}\text { Canada } \\
n_{1}=63\end{array}$ & $\begin{array}{c}\text { États-Unis } \\
n_{2}=74\end{array}$ & $\mathbf{p}^{2}$ & $\begin{array}{c}\text { Canada } \\
n_{3}=69\end{array}$ & $\begin{array}{c}\text { États-Unis } \\
n_{4}=67\end{array}$ & $\mathbf{p}^{2}$ \\
\hline \multicolumn{7}{|l|}{ Compétences technologiques } \\
\hline $\begin{array}{l}\text { investissement } \\
\text { en R-D (\%) }\end{array}$ & 3,60 & 2,30 & & 5,96 & 2,70 & $* * *$ \\
\hline $\begin{array}{l}\text { veille technologique } \\
\text { adoption de technologies }\end{array}$ & 5,10 & 4,92 & & 5,44 & 4,95 & $* * *$ \\
\hline $\begin{array}{l}\text { avancées } \\
\text { compétences techniques }^{4}\end{array}$ & 4,57 & 6,57 & $* * *$ & 8,06 & 9,81 & $* * *$ \\
\hline des employés ${ }^{3}$ & 5,14 & 5,18 & & 5,52 & 5,13 & $*$ \\
\hline $\begin{array}{l}\text { savoir-faire unique } \\
\text { lié aux produits }{ }^{3}\end{array}$ & 4,80 & 5,11 & & 4,98 & 5,51 & * \\
\hline \multicolumn{7}{|c|}{ Compétences organisationnelles } \\
\hline habiletés de gestion ${ }^{3}$ & 4,55 & 5,13 & $* *$ & 5,14 & 5,26 & \\
\hline efforts de marketing ${ }^{3}$ & 3,67 & 4,10 & & 4,70 & 4,39 & \\
\hline $\begin{array}{l}\text { stabilité financière }{ }^{3} \\
\text { degré d'internationalisation }\end{array}$ & 4,95 & 4,82 & & 4,84 & 4,89 & \\
\hline des ventes $(\%)$ & 24,39 & 8,63 & $* * *$ & 51,63 & 12,66 & $* * *$ \\
\hline $\begin{array}{l}\text { réputation }{ }^{3} \\
\text { stabilité des réseaux }\end{array}$ & 5,86 & 5,90 & & 5,87 & 5,90 & \\
\hline fournisseurs et clients ${ }^{3}$ & 5,06 & 4,80 & & 5,09 & 5,00 & \\
\hline \multicolumn{7}{|c|}{ Caractéristiques de la relation } \\
\hline degré de dépendance (\%) & 34,9 & 41,9 & & 15,9 & 32,8 & $* *$ \\
\hline degré d'exigence 3 & 4,47 & 4,11 & $*$ & 4,71 & 4,30 & $* *$ \\
\hline degré d'influence 3 & 3,11 & 2,64 & $* *$ & 2,90 & 3,25 & $*$ \\
\hline
\end{tabular}

1. Le nombre d'employés moyen pour les deux pays est le suivant: les firmes de petite taille, 21 et 18 respectivement; les firmes de taille moyenne, 156 et 148 respectivement.

2. Niveau de signification établi par le test de Student :

${ }^{*} \mathrm{p}<0,10 ;{ }^{* *} \mathrm{p}<0,05 ;{ }^{* * *} \mathrm{p}<0,01 ;{ }^{* * * *} \mathrm{p}<0,001$; test bilatéral.

3. Mesures perceptuelles établies à l'aide d'échelles Likert en sept points; voir annexe.

4. Voir figure 1 pour la liste des technologies.

5. Voir Bourgault (1996) pour une évaluation détaillée des différences basées strictement sur la taille (sans égard à la localisation géographique). 
En ce qui touche les firmes de petite taille, on constate principalement qu'il existe peu de différences significatives entre les compétences canadiennes et américaines. Les principales différences recoupent celles déjà identifiées, soit la capacité d'exporter des firmes canadiennes et le nombre de technologies de pointe utilisées par les firmes américaines. Les taux de dépendance, même s'ils ne présentent pas de différences significatives, sont relativement élevés dans les deux cas et témoignent d'une situation critique pour ce qui est des relations des firmes avec leurs clients. L'autonomie limitée de ces dernières est, du reste, typique des firmes de petite taille, qu'il s'agisse d'ateliers traditionnels (machine shops) ou de firmes de haute technologie dont la gamme de produits est peu étendue.

L'analyse des firmes de taille moyenne révèle des différences plus marquées entre les deux sous-groupes, en particulier sur le plan technologique. En fait, le profil de différences entre les deux pays se rapproche beaucoup de la première analyse faite à partir de tout l'échantillon (tableau 2), sauf pour le degré d'influence dont la valeur est plus réduite pour les firmes canadiennes.

\section{Compétences et performance des sous-traitants}

Une première analyse a mené à l'évaluation des déterminants de la performance sur une base géographique. Le tableau 4 présente les variables qui démontrent le plus fort pouvoir explicatif sur la variable dépendante.

Les principaux facteurs explicatifs de la performance des firmes canadiennes sont les compétences techniques des employés, la réputation de l'entreprise, l'intensité en R-D et les habiletés de gestion. Sur le plan technologique, ce résultat confirme l'apport important d'une main-d'œuvre spécialisée et de la valeur des activités d'innovation dans une industrie comme l'aérospatiale. Le résultat hautement significatif pour la réputation autorise à penser que le niveau élevé des exigences des donneurs d'ordres favorise les firmes expérimentées et expose, à l'inverse, les non initiées à davantage de risques. Les habiletés de gestion constituent aussi un fort déterminant de la performance des sous-traitants. Le rôle du dirigeant dans le succès d'une firme, du point de vue des orientations générales comme de l'acquisition et de l'utilisation des ressources, revêt une importance stratégique au sein d'une industrie en constante mutation (fusions, réduction du nombre de soustraitants, etc.). L'importance du dynamisme managérial a été vérifiée dans plusieurs industries, et en particulier dans l'aérospatiale et la défense (O'Guin, 1995).

Le degré d'internationalisation des ventes, pris ici comme une mesure de la capacité des firmes à être compétitives sur les marchés internationaux, est lié négativement à la performance des firmes canadiennes. Ce résultat est pour le moins surprenant et laisse croire que les activités d'exportation ne sont pas suffisamment orientées vers des produits ou marchés stratégiques. Le degré d'exigence des donneurs d'ordres perçu par le sous-traitant est également lié de façon négative à 
TABLEAU 4

Variables explicatives de la performance selon la situation géographique

\begin{tabular}{|c|c|c|}
\hline & $\begin{array}{l}\text { Canada } \\
n_{1}=132\end{array}$ & $\begin{array}{c}\text { États-Unis } \\
n_{2}=141\end{array}$ \\
\hline \multicolumn{3}{|l|}{ Compétences technologiques } \\
\hline $\begin{array}{l}\text { intensité en R-D } \\
\text { veille technologique } \\
\text { adoption de technologies avancées } \\
\text { compétences techniques } \\
\text { savoir-faire unique }\end{array}$ & $\begin{array}{l}0,24 * * * \\
0,32 * * *\end{array}$ & $0,17 * *$ \\
\hline \multicolumn{3}{|l|}{ Compétences organisationnelles } \\
\hline $\begin{array}{l}\text { habiletés de gestion } \\
\text { efforts en marketing } \\
\text { stabilité financière } \\
\text { internationalisation des ventes } \\
\text { réputation } \\
\text { stabilité des réseaux }\end{array}$ & $\begin{array}{c}-0,32 * * * \\
0,31 * * * *\end{array}$ & $\begin{array}{l}0,42^{* * * *} \\
0,24 * * * \\
0,16^{*}\end{array}$ \\
\hline \multicolumn{3}{|l|}{ Variables contextuelles } \\
\hline $\begin{array}{l}\text { degré de dépendance } \\
\text { degré d'exigence } \\
\text { degré d'influence } \\
\text { taille de la firme sous-traitante }\end{array}$ & $\begin{array}{l}-0,23 * * * \\
-0,14^{*}\end{array}$ & $\begin{array}{l}-0,22 * * \\
-0,20 * * * \\
-0,22 * * *\end{array}$ \\
\hline $\mathbf{R}^{2}$ & $57,5 \% * * * *$ & $48,0 \% * * * *$ \\
\hline
\end{tabular}

1. Niveau de signification : ${ }^{*} \mathrm{p}<0,10 ;{ }^{* *} \mathrm{p}<0,05 ;{ }^{* * *} \mathrm{p}<0,01 ;{ }^{* * * *} \mathrm{p}<0,001$.

sa performance. Un coefficient négatif dans la régression démontre une faiblesse entre la perception des besoins de la clientèle et la nécessité d'y répondre. Une firme dont les dirigeants trouvent difficile de prévoir les besoins des clients et de s'y ajuster s'avère donc une firme moins bien adaptée pour faire face aux conditions du marché : elle tendra à se positionner moins bien au regard des critères de performance.

Les sous-traitants américains présentent, quant à eux, un profil différent. Lorsque l'on considère l'ensemble des variables, les habiletés de gestion représentent un déterminant très important suivi de la stabilité financière. Habiletés de gestion et stabilité financière sont de fait complémentaires et toutes deux indispensables pour des entreprises qui, évoluant dans un environnement aussi tumultueux que celui de l'industrie américaine des cinq dernières années, doivent faire preuve de leadership et de rigueur dans la gestion des ressources.

Les résultats révèlent aussi des facteurs explicatifs intéressants sur le plan des compétences technologiques : veille technologique et savoir-faire unique ressortent en effet de façon significative. Ces deux caractéristiques permettent de tracer un 
profil technologique plutôt différent de celui des sous-traitants canadiens. Les firmes américaines visent le développement d'une certaine spécificité sur le plan technologique, tout en étant à l'affût des innovations techniques et des marchés émergents. Au comportement proactif qui les caractérise s'ajoute la relative autonomie dont elles font preuve : on observe en effet que degré d'exigence et degré d'influence des donneurs d'ordres sont tous les deux affectés d'un coefficient négatif. Les PME sous-traitantes tiennent à leur indépendance et, contrairement à la tendance au partenariat véhiculée par le discours dominant, maintiennent une certaine distance entre eux et leurs clients.

\section{Discussion}

Les déterminants de la performance sont donc différents d'un pays à l'autre, ce qui peut avoir un impact sur la capacité des PME à être actives sur les marchés du pays voisin. Le sous-traitant canadien désireux de s'introduire dans les marchés américains aurait donc intérêt à se pencher sur les règles américaines. Ce fait est d'autant plus pertinent qu'il existe effectivement des différences significatives entre les deux pays, comme l'ont montré les statistiques descriptives (tableau 2). Au regard de l'adoption des technologies, par exemple, les firmes américaines ont un profil plus avancé que les firmes canadiennes.

Ces résultats soulèvent plusieurs questions si l'on considère que les grands donneurs d'ordres sont de moins en moins confinés à leur territoire national (ex. : Boeing possède des usines au Canada et Bombardier a des usines aux ÉtatsUnis) et qu'ils semblent tenir peu compte de la situation géographique des soustraitants qu'ils choisissent. Les différences entre les deux pays peuvent aussi être le reflet d'une différence dans l'allocation des contrats. En effet, on peut supposer qu'à long terme les facteurs associés à la performance des sous-traitants dépendront de la nature des travaux exigés par les donneurs d'ordres. Est-ce à dire que les types de contrats accordés aux sous-traitants canadiens sont différents de ceux accordés aux Américains ? Ces questions se posent à la lumière des résultats obtenus. Les sous-traitants canadiens eux-mêmes, et en particulier les plus petits, ont déjà soulevé la question de l'allocation des contrats (MacNamara, 1989).

Pour les firmes canadiennes, cette situation constitue donc un défi énorme. Il faut certes exceller sur le plan de la production, mais ce n'est plus suffisant dans les conditions actuelles ; même à ce niveau, les sous-traitants canadiens sont en retard sur leurs concurrents américains. Étant donné l'étroitesse du marché intérieur, les firmes doivent redoubler d'efforts pour développer des spécialités. Mais les fruits de leur démarche dépendent souvent de la situation dans laquelle elles se trouvent. Une firme de petite taille peut-elle atteindre le niveau d'excellence qu'elle espère ? Oui, si l'on se fie aux données du tableau 4, puisque dans chacun des sous-groupes (Canada et États-Unis), le coefficient obtenu par la variable taille est de signe négatif. 
Pour répondre de façon plus précise à cette dernière question, une dernière analyse a été réalisée à partir des deux sous-groupes décrits plus tôt sur la base de la taille médiane. Les résultats des régressions (non illustrés) montrent que pour les petites firmes canadiennes, l'ensemble des variables explicatives les plus importantes confirme le portrait traditionnel de l'entreprise axée vers l'exécution de tâches définies par le donneur d'ordres : compétences techniques doublées de capacités de production et lien étroit entretenu avec le client. La performance des petites firmes américaines est, quant à elle, largement expliquée par le savoir-faire unique et les habiletés de gestion. Ajoutées à une relative aversion pour la dépendance, ces dimensions semblent établir le profil de la PME innovante qui tente de développer une certaine autonomie sur les marchés.

Chez les firmes de taille moyenne, les déterminants de la performance montrent des écarts également marqués entre les firmes canadiennes et américaines. Au Canada, le profil des firmes de taille moyenne diffère peu de celui de l'ensemble des firmes. Du côté américain, la réputation, la stabilité financière et les habiletés de gestion priment du côté organisationnel alors que l'adoption de technologies de production et les activités de veille représentent d'importants déterminants sur le plan technologique.

\section{Conclusion}

Prise dans son ensemble, l'industrie aérospatiale canadienne se distingue par son incontestable compétitivité. Depuis 15 ans, elle a connu un rythme de croissance qui dépasse celui de ses principales rivales étrangères. Ce succès, qui est régulièrement mis de l'avant par les gouvernements et les associations industrielles, s'explique essentiellement par la performance de quelques grandes entreprises. La présente recherche a voulu vérifier dans quelle mesure ce succès était aussi celui des PME sous-traitantes canadiennes œuvrant dans le secteur.

Le développement de l'industrie aérospatiale canadienne est intimement lié à celui de sa correspondante américaine. La proximité géographique, les politiques de libre-échange, la présence de grandes entreprises de part et d'autre de la frontière facilitent l'attribution des contrats de sous-traitance d'un pays vers l'autre. Les PME canadiennes se trouvent donc à concurrencer, dans une large mesure, les soustraitants américains et c'est pourquoi elles devraient adopter des pratiques leur permettant d'obtenir leur part de contrats. Ce défi est amplifié par le fait que le plus important producteur au monde (les États-Unis) est également celui qui assure le mieux son autosuffisance sur une base industrielle. Au Canada, les liens intraindustriels ne sont pas aussi solides, comme le constatent les autorités gouvernementales (Industrie Canada, 1995). 
L'étude montre toutefois des différences importantes entre les PME soustraitantes des deux pays. Le retard des firmes canadiennes sur le plan technologique apparaît nettement. Et s'il peut exister des firmes réussissant à développer des compétences spécifiques, l'ensemble des sous-traitants souffre d'un manque de dynamisme dans la création et le développement. Devant des phénomènes irréversibles comme la croissance des pays asiatiques et la diminution de la base de soustraitance, les firmes doivent réagir promptement. Les écarts sont davantage accentués pour les firmes de taille moyenne, ce qui permet de conclure à une croissance plus forte des sous-traitants américains. Ces derniers se démarquent notamment par l'éventail des technologies avancées qu'ils adoptent. S'engager dans une industrie comme l'aérospatiale exige des PME sous-traitantes de multiples efforts, puisqu'elles doivent rapidement affronter une vive concurrence. Cette réalité est d'autant plus vraie au Canada que la dimension du marché national y est réduite. Le marché américain est certainement le plus accessible pour les firmes canadiennes à condition qu'elles adoptent un profil technologique et organisationnel correspondant à ses exigences. La présente recherche a montré que l'écart entre les deux pays est encore très grand. Les PME canadiennes semblent évoluer moins rapidement et conservent encore souvent un profil de sous-traitance traditionnel. Le passage vers un profil de classe supérieure, aussi souhaitable qu'il soit, demeure long et difficile. La présente étude en repère néanmoins les principaux jalons, en tenant compte du contexte nord-américain.

D'un point de vue plus global, les différences observées entre les soustraitants canadiens et américains soulèvent des questions cruciales pour l'avenir de l'industrie. Compte tenu du contexte canadien, les grandes entreprises nationales peuvent-elles se développer à long terme sans la présence de sous-traitants nationaux concurrentiels? Quel rôle peuvent jouer les donneurs d'ordres dans l'amélioration de la qualité de la sous-traitance ? De quelle nature doivent être les politiques industrielles pour accroître cette qualité ? Ces questions demeurent ouvertes à l'analyse et devraient préoccuper l'ensemble des intervenants soucieux de maintenir le degré de performance actuel de cette industrie. Par ailleurs, l'étude de l'industrie canadienne présente un intérêt certain pour les pays dont le secteur aérospatial, bien que modeste, est à l'heure actuelle en phase de développement. 


\section{Annexe Variables utilisées}

\section{Performance (4 éléments) Définition et mesure opérationnelle}

- qualité du produit et du service

- délais de livraison

- flexibilité

- coût
- perception de la qualité des produits comme constituant un atout pour l'entreprise, par comparaison aux plus proches concurrents (échelle de Likert en 7 points)

- perception des délais de livraison comme constituant un atout pour l'entreprise, par comparaison aux plus proches concurrents (échelle de Likert en 7 points)

- perception de la flexibilité de la production comme constituant un atout pour l'entreprise, par comparaison aux plus proches concurrents (échelle de Likert en 7 points)

- perception du prix des produits comme constituant un atout pour l'entreprise, par comparaison aux plus proches concurrents (échelle de Likert en 7 points)
Variables mesurant les compétences

- investissement en R-D

- adoption de technologies avancées

- veille technologique

- compétences techniques des employés

- savoir-faire unique lié aux produits

- habiletés de gestion

- efforts de marketing

- stabilité financière

- degré d'internationalisation des ventes

\section{Définition et mesure opérationnelle}

- mesure factuelle : ratio des sommes allouées en R-D sur les ventes annuelles

- mesure factuelle évaluant le nombre de technologies adoptées selon les trois catégories suivantes : les technologies informatisées de gestion, les technologies informatisées pour la conception/fabrication et les programmes d'amélioration de production (cf. figure 1 pour liste)

- perception du degré de connaissance des nouveaux développements technologiques : produits, matériaux et procédés (échelle de Likert en 7 points)

- perception du degré de connaissance de la disponibilité commerciale des nouvelles technologies de l'information et de production (échelle de Likert en 7 points)

- perception du degré de connaissance des avantages comparatifs découlant de l'utilisation de ces nouvelles technologies (échelle de Likert en 7 points)

- perception des habiletés techniques des employés comme constituant un atout pour la firme (échelle de Likert en 7 points)

- perception du savoir-faire unique et exclusif par rapport au produit, comme constituant un atout pour la firme (échelle de Likert en 7 points)

- perception des habiletés de gestion comme constituant un atout principal pour la firme (échelle de Likert en 7 points)

- perception des efforts en marketing comme constituant un atout principal pour la firme (échelle de Likert en 7 points)

- perception de la stabilité financière comme étant un atout principal pour la firme (échelle de Likert en 7 points)

- pourcentage des ventes réalisées à l'extérieur du pays 


\section{Annexe \\ Variables utilisées (suite)}

- réputation

- efforts de maintien des réseaux avec l'externe

- degré de dépendance du sous-traitant

- degré d'influence du donneur d'ordres

- degré d'exigence du donneur d'ordres
- perception de la réputation comme constituant un atout principal pour la firme (échelle de Likert en 7 points)

- perception de la stabilité du réseau de fournisseurs (échelle de Likert en 7 points)

- perception de la stabilité du réseau de clients fournisseurs (échelle de Likert en 7 points)

- pourcentage du chiffres d'affaires réalisé avec un nombre restreint de clients (seuil de dépendance fixé à $25 \%$ )

- degré d'influence sur les décisions d'adoption de nouvelles technologies (échelle de Likert en 7 points)

- degré d'influence sur le choix des employés de production (échelle de Likert en 7 points)

- degré d'influence sur la formation des employés de production (échelle de Likert en 7 points)

- degré d'implication financière sur la modernisation des activités de production (échelle de Likert en 7 points)

- perception du degré de facilité de prédiction des exigences du donneur d'ordres (échelle de Likert en 7 points)

- perception du degré de variabilité des exigences du donneur d'ordres (échelle de Likert en 7 points)

- perception de l'ampleur des ajustements nécessaires par rapport aux exigences changeantes (échelle de Likert en 7 points)

- perception de la durée d'adaptation nécessaire pour répondre aux exigences changeantes (échelle de Likert en 7 points)

\section{Bibliographie}

AEROSPACE INDUSTRIES ASSOCIATION of AMERICA (1997), « Aerospace, facts \& figures 1996-1997 », Washington, D.C., The Aerospace Research Center.

BERGERON, F. et L. RAYMOND (1996), «EDI dans la PME et la grande entreprise : similitudes et différences », Revue internationale PME, vol. 9, $\mathrm{n}^{\circ}$ 1, p. 40-60.

BESSANT, J. (1993), «The lessons of failure : learning to manage new manufacturing technology », International Journal of Technology Management, vol. 8, nos 2/3/4, p. 197-215.

BLENKHORN, D. L. et A.H. NoORI (1990). «What it takes to supply Japanese OEMs », Industrial Marketing Management, vol. 19, p. 21-30.

BONACCORSI, A. (1992), «On the relationship between firm size and export intensity», Journal of International Business Studies, vol. 23, no 4, p. 605-635.

BOURGAULT, M. (1996), Compétences des entreprises et sous-traitance nord-américaine : le cas de l'industrie aérospatiale, Thèse de doctorat (Ph.D.), École polytechnique de Montréal.

DE BRENTANI, U. (1989), «Success and failure in new industrial services », Journal of Production Innovation Management, vol. 6, p. 239-258. 
DeBresson, C., J. Niosi, R. Dalpé et D. Wine (1991), «Liaisons technologiques et contrôle étranger dans l'industrie aéronautique canadienne », dans D. McFetridge, (éd.), Investissement étranger, technologie et croissance économique, Calgary, The University of Calgary Press et Ottawa, Approvisionnements et Services Canada.

DEMERS, C. et T. HAFSI (1993), «Compétitivité et nation : jeux dominants et jeux périphériques », Gestion, septembre, p. 48-65.

Doz, Y. (1994), «Les dilemmes de la gestion du renouvellement des compétences clés », Revue française de gestion, janvier-février, p..92-104.

DunN, D.T. Jr, J.H. FrIAR et C.A. ThOMAS (1991), «An approach to selling high-tech solutions », Industrial Marketing Management, vol. 20, p. 149-159.

DYER, J.H. et W.G. OUCHI (1993), «Japanese-style partnerships : giving companies a competitive edge », Sloan Management Review, automne, p. 51-63.

FUNK, J.L. (1993), «Japanese product-development strategies : a summary and propositions about their implementation ", IEEE Transactions on Engineering Management, vol. 40, n 3, p. 224-236.

HAHN, C.K., C.A. WATTS et K.Y. KIM (1990), "The supplier development program : a conceptual model », Journal of Purchasing and Materials Management, printemps, p. 2-20.

HALL, R. (1993), «A framework linking intangible resources and capabilities to sustainable competitive advantage», Strategic Management Journal, vol. 14, p. 607-618.

HAN, S., D.T. WILSON et S.P. DANT (1993), «Buyer-supplier relationships today », Industrial Marketing Management, vol. 22, p. 331-338.

HELPER, S.R. et M. SAKo (1995), «Supplier relations in Japan and the United States : are they converging ?», Sloan Management Review, printemps, p. 77-84.

HELPER, S.R. (1991), « How much has really changed between U.S. automakers and their suppliers », Sloan Management Review, été, p. 15-28.

INDUSTRIE CANADA (1995), Canada's Aircraft Industry - Background Analysis. Direction générale de l'aéronautique et de la défense, Gouvernement du Canada, Ottawa.

INDUSTRIE CANADA (1996a), Aerospace and Defence-Related Industries : Statistical Survey Report - 1995, Direction générale de l'aéronautique et de la défense, Gouvernement du Canada, Ottawa.

Industrie CANAda (1996b), Canada's International Business Strategy 1996-1997: Aerospace and Defence (no 2), Ministère des Approvisionnements et Services, cat. $\mathrm{n}^{\circ} \mathrm{C} 2-226 / 2-1996 \mathrm{E}$, Ottawa.

JACOB, R., P.A. JULIEN et L. RAYMOND (1997), «Compétitivité, savoirs stratégiques et innovation : les leviers de l'apprentissage collectif en contexte de réseau ", Gestion, vol. 22, no 3, p. 93-100.

JACOBS, D. et M.W. DE JONG (1992), «Industrial clusters and the competitiveness of the Netherlands : empirical results and conceptual issues », The Economist, $\mathrm{n}^{0} 2$, p. 233-252.

JULIEN, P.A. et collaborateurs (1997), Les PME : Bilan et perspectives, $2^{\mathrm{e}}$ éd., Cap-Rouge (Québec), Presses Inter Universitaires. 
KAMATH, R.R. et J.K. LIKER (1990), «Supplier dependence and innovation : a contingency model of suppliers' Innovative activities », Journal of Engineering and Technology Management, vol. 7, p. 111-127.

KAMATH, R.R. et J.K. LIKER (1994), «A second look at Japanese product development », Harvard Business Review, vol. 72, novembre-décembre, p. 154-170.

KOLAY, M.K. (1992), « Suppliers asset base : appreciating or depreciating ?», International Journal of Operations and Production Management, vol. 13, no 8, p. 72-86.

Lefebvre, L. A., J. HARVEY et E. LefEbVRE (1991), « Technological experience and the technology adoption decisions in small manufacturing firms ", R\&D Management, vol. 21, no 3, p. 241-249.

Lefebvre, E., L.A. Lefebvre et M.J. Roy (1995), «Technological penetration and organizational learning in SMEs : the cumulative effect », Technovation, vol. 15, $\mathrm{n}^{\circ} 8$, p. 511-522.

LiKER, J.K., R.R. KAMATH, S.N. WASTI et M. NAGAMASHI (1996), « Supplier involvement in automotive component design : are there really large US Japan differences?», Research Policy, vol. 25, p. 59-89.

MACNAmARA (1989), Study of the Aerospace Machine Shops in Canada, rapport préparé pour la Division des firmes spécialisées, Direction de l'aéronautique, de la défense et des retombées industrielles, Ministère de l'Industrie, de la Science et de la Technologie, Ottawa.

MEREDITH, J. (1988), « The role of manufacturing technology competitiveness : peerless laser processors », IEEE Transactions on Engineering Management, vol. 35, $\mathrm{n}^{\circ} 1$, p. 3-10.

MORBEY, G.K. (1988), «R\&D : its relationship to company performance », Journal of Product Innovation Management, vol. 5, p. 191-200.

OAKEY, R. (1994), New Technology-Based Firms in the 1990s, Londres, Paul Chapman Publishing Ltd.

OCDE - ORGANISATION POUR LA COOPÉRATION ET LE DÉVELOPPEMENT ÉCONOMIQUE (1995), Études économiques de l'OCDE 1994-1995, Paris.

O'Guin, M. (1995), «Aerospace and defense contractors learn how to make their businesses soar », Quality Progress, juin, p. 35-42.

ÖSTERLUND, J. (1991), «The resource box; competence management by informatics in high technology R\&D work», IEEE Transactions on Engineering Management, vol. $38, \mathrm{n}^{\circ} 2$, p. 164-170.

PALIWODA, S. J. et A. J. BONACCORSI (1993), «Systems selling in the aircraft industry », Industrial Marketing Management, vol. 22, p. 155-160.

PorTER, M.E. (1990), The Competitive Advantage of Nations, New York, Free Press.

PRESUTTI, W.D. Jr. (1991), «Technology management : an important element in the supplier capability survey», International Journal of Purchasing and Materials Management, hiver, p. 11-15. 
Rugman, A.M. et J.R. O'CRUZ (1993), «The double diamond model of international competitiveness : the Canadian experience », Management International Review, vol. 33, numéro spécial, p. 17-40.

SAViotTI, P.P. (1995), « Renouveau des politiques industrielles : le point de vue des théories évolutionnistes ", Revue d'économie industrielle, vol. 71, $1^{\mathrm{er}}$ trimestre, p. 199-212.

SCHEWE, G. (1994), «Successful innovation management : an integrative perspective», Journal of Engineering and Technology Managment, vol. 11, p. 25-53.

SEN, F. et A.H. RUBENSTEIN (1990), «An exploration of factors affecting the integration of in-house R\&D with external technology acquisition strategies of a firm », IEEE Transactions on Engineering Management, vol. 37, $\mathrm{n}^{\circ} 4$, p. 246-258.

STATISTIQUe CANADA (1995), Recherche et développement industriels, catalogue $n^{\circ}$ 88-202, Ministère de l'Industrie, des Sciences et de la Technologie, Gouvernement du Canada, Ottawa.

StatistiQue CANAda (1989), Survey of Manufacturing Technologies, Statistical tables, Ministère de l'Industrie, des Sciences et de la Technologie, Service des approvisionnements, Gouvernement du Canada, Ottawa.

STUART, F.I. et D. MCCUTCHEON (1995), « Problem sources in establishing strategic supplier alliances », International Journal of Purchasing and Materials Management, hiver, p. 3-9.

Swamidass, P.M. (1994), Technology on the Factory Floor II, Washington, The Manufacturing Institute.

VAN WYK, R. (1997), «Strategic technology scanning », Technological Forecasting and Social Change, vol. 55, p. 21-38.

ZIRGER, B.J. et M. MAIDIQUE (1990), « A model of new product development : an empirical test », Management Science, vol. 36, nº 7, p. 867-883. 